\title{
Grid turbulence in dilute polymer solutions: PEO in water
}

\author{
Richard Vonlanthen ${ }^{1}$ and Peter A. Monkewitz ${ }^{2, \dagger}$ \\ ${ }^{1}$ Bühler Group, CH-9240 Uzwil, Switzerland \\ ${ }^{2}$ Swiss Federal Institute of Technology (EPFL), CH-1015 Lausanne, Switzerland
}

(Received 16 November 2012; revised 3 May 2013; accepted 13 June 2013; first published online 30 July 2013)

Grid turbulence of polyethylene oxide (PEO) solutions (Polyox WSR-301 in $\mathrm{H}_{2} \mathrm{O}$ ) has been investigated experimentally for three concentrations of 25,50 and 100 weight ppm, at a turbulence Reynolds number based on a Taylor microscale of $\operatorname{Re}_{\lambda} \approx 100$. For the first time, time sequences of turbulence spectra have been acquired at a rate of $0.005 \mathrm{~Hz}$ to reveal the spectral evolution due to mechanical degradation of the polymers. In contrast to spectra averaged over the entire degradation process, the sequence of spectra reveals a clear but time-dependent Lumley scale at which the energy spectrum changes abruptly from the Kolmogorov $\kappa^{-5 / 3}$ inertial range to a $\kappa^{-3}$ elastic range, in which the rate of strain is maintained constant by the polymers. The scaling of the initial Lumley length with Kolmogorov dissipation rate $\epsilon_{0}$ and molecular weight is determined, and a cascade model for the temporal decrease of molecular weight, i.e. for the breaking of polymer chains is presented. Finally, a heuristic model spectrum is developed which covers the cases of both maximum and partial turbulence reduction by polymers.

Key words: homogeneous turbulence, viscoelasticity

\section{Introduction}

The Toms effect (Toms 1948) describes a phenomenon where the addition of minute amounts of polymer, of the order of a few parts per million by weight (wppm), of high molar mass allows the skin friction to be lowered significantly in wall-bounded turbulent flows. In addition to their obvious industrial relevance, these drag-reducing flows also present a fundamental interest (see e.g. Lumley 1969; Virk 1975; Hinch \& Elata 1979; Sellin et al. 1982; Sureshkumar, Beris \& Handler 1997; Sreenivasan \& White 2000; Balkovsky, Fouxon \& Lebedev 2001; De Angelis, Casciola \& Piva 2002; Fouxon \& Lebedev 2003; Benzi et al. 2003; De Angelis et al. 2005; Berti et al. 2006; Brostow 2008; Ouellette, Xu \& Bodenschatz 2009). We emphasize immediately that the present study is concerned exclusively with high-Reynolds-number turbulence and not with the low-Reynolds-number structural turbulence (McComb 1990), also called elastic turbulence (Groisman \& Steinberg 2000, 2004).

Since Toms' discovery, a vast number of experimental, numerical and analytical efforts have been made to understand and model the interaction between turbulence 
and polymers, with only partial success so far. In this study we limit ourselves to the study of dilute solutions of polyethylene oxide (PEO; Polyox WSR-301 with nominal molar weight of $4 \times 10^{6} \mathrm{~g} \mathrm{~mol}^{-1}$ ) in water, where 'dilute' means that there is no or insignificant entanglement between individual polymer chains, even when extended. In the present experiments the size of extended polymer chains is about an order of magnitude smaller than the Kolmogorov scale characterizing the smallest turbulent eddies. Hence, there are of the order of $10^{3}$ polymer molecules in the smallest eddy, even at the lowest PEO concentration of $25 \mathrm{wppm}$, so that the effect of polymers can be modelled by modified bulk properties of the working fluid. In this regime, the interaction between turbulence and individual polymer chains is conceptually simple. It has been very well-summarized by Balkovsky et al. (2001) so that we just quote here from page 056301-8 of their paper:

\begin{abstract}
'Whereas in a pure solvent, typical gradients of the velocity grow unlimited as the Reynolds number increases, in polymer solutions the balance of inertial and elastic degrees of freedom fixes the characteristic value of the gradient at $1 / \tau$. [ $\tau$ represents the polymer relaxation time.] Indeed, if the instantaneous velocity gradient exceeds $1 / \tau$, it extends the polymers, so that the elastic stress grows and damps the gradient. On the other hand, if the velocity gradient is much smaller than $1 / \tau$, the molecules contract and do not produce any effect on the flow. Then the velocity gradients tend to grow to the value characteristic of the pure solvent, which is larger than $1 / \tau$ above the transition'.
\end{abstract}

The majority of existing experimental studies on turbulence in polymer solutions were carried out in pipe flows (see, e.g., Virk et al. 1967; Patterson, Zakin \& Rodrigue 1969; Den Toonder et al. 1997; Escudier, Presti \& Smith 1999; Warholic, Massah \& Hanratty 1999; Kim \& Sirviente 2007), with only a few investigations in grid-generated turbulence (Gadd 1965; Barnard \& Sellin 1969; Friehe \& Schwarz 1970; McComb, Allan \& Greated 1977; van Doorn, White \& Sreenivasan 1999) or other 'washing-machine-like' configurations producing nearly homogeneous and isotropic turbulence (Liberzon et al. 2006, 2009). The difference between the effect of polymers on turbulence without and with mean shear is that in the former case the polymers can only provoke additional local energy dissipation, while in the latter case they can in addition modify the mean shear and with it long-range energy exchange by instabilities. While the early investigations of grid turbulence or similar arrangements showed a smooth gradual reduction of spectral energy density at increasing wavenumbers, Berti et al. (2006) appears to have been the first to find in his numerical simulation a distinct scale beyond which turbulent energy is reduced by the polymers. As this behaviour has been anticipated by Lumley (1964) (see also Lumley 1969, 1973; Hinch 1977; Hinch \& Elata 1979, and others), this scale will be called here the 'Lumley scale'. The reason why this Lumley scale has not been clearly evident from previous experimental turbulence spectra was spectral averaging over times that were too long compared with the characteristic time for significant mechanical degradation (scission) of polymer molecules. This is demonstrated by the present investigation in which the averaging time of turbulence spectra has been reduced to a minimum in order to obtain 10 consecutive spectra over the duration of an experimental run $(\sim 34 \mathrm{~min})$ which clearly reveal the nature of 'instantaneous' turbulence spectra minimally affected by polymer degradation.

The paper is organized as follows. In $\S 2$ we describe the experimental and particle image velocimetry (PIV) set-ups used to acquire turbulence spectra. It also includes a detailed description of the PIV data processing, crucial for the quality of the results, and a description of how the turbulence was characterized. The following $\S 3$ is 
devoted to the characterization of the PEO solutions, in particular their molar weight and viscosities. This leads to the presentation of the central results: the construction of a model spectrum in $\S 4$ to match our measurements, the experimental turbulence spectra in $\S 5$ and the extraction of the time-dependent Lumley length and other global turbulence characteristics from the data in $\S 6$. The latter section also includes the formulation of a 'cascade model' for the temporal decrease of molar weight due to mechanical breakage of PEO molecules. The paper concludes with an outlook in $§ 7$.

\section{Experimental set-up}

\subsection{Facility}

The facility for the present investigation is a closed-loop hydraulic tunnel with a centreline length of $11.4 \mathrm{~m}$ and a capacity of roughly 36001 (see figure 1). Part of the return section of $600 \mathrm{~mm} \times 600 \mathrm{~mm}$ cross-section has an open surface to allow the in situ mixing of PEO with water. The leg containing the test section contains a $600 \mathrm{~mm} \times 600 \mathrm{~mm}$ honeycomb followed by $600 \mathrm{~mm}$ of straight section, a 12:1 contraction and the turbulence grid. The square grid with a mesh size of $M=16 \mathrm{~mm}$ is made of $3 \mathrm{~mm}$ round rods. Tethered beads of $12 \mathrm{~mm}$ diameter are attached at each grid node and approximately double the turbulence production relative to the bare grid. This enhanced turbulence grid is the 'ETG' grid described in detail in Vonlanthen \& Monkewitz (2011). Downstream of the grid is the closed test section of $900 \mathrm{~mm}$ length with a $200 \mathrm{~mm} \times 150 \mathrm{~mm}$ cross-section (see figure 1 for details). Note that the test section is straight, without a secondary contraction sometimes used in grid turbulence experiments (see again Vonlanthen \& Monkewitz 2011).

A homemade bladeless disc pump, also called a Tesla pump, similar to that described by Den Toonder et al. (1995) is located downstream of the test section. The fluid enters the pump axially through a honeycomb which avoids transmitting fluid rotation upstream into the test section and is entrained by friction on a stack of smooth rotating annular discs oriented normal to the axis. This pump minimizes the mechanical destruction of polymer chains and is capable of producing a mean velocity $U_{0}$ (equal to the volume flow rate, monitored by the pressure drop across the contraction, divided by the cross-sectional area) of up to $10^{3} \mathrm{~cm} \mathrm{~s}^{-1}$. However, all experiments presented here have been carried out at a fixed $U_{0}=120 \pm 5 \mathrm{~cm} \mathrm{~s}^{-1}$ in order to limit the rate of polymer degradation. This mean velocity corresponds to a grid mesh Reynolds number $U_{0} M / \nu$ of approximately $2 \times 10^{4}$. For more details on the flow facility the reader is referred to the theses of Pipe (2005) and Vonlanthen (2010).

\subsection{PIV set-up}

Velocity measurements in the PEO solutions were carried out with PIV (see e.g. Raffel et al. 2007). Illumination was provided by a twin Nd:YAG laser $(15 \mathrm{~mJ}$ per pulse at $532 \mathrm{~nm}$, Quantel Brilliant Twins B). A standard stereo set-up with two 11 megapixel $(4008 \times 2672$ pixels) double-buffer cameras (TSI PowerView Plus) with $60 \mathrm{~mm} f / 2.8$ lenses was used but the quality of the out-of-plane velocity component $u_{3}$ finally turned out to be unsatisfactory in comparison with the in-plane components $u_{1}$ and $u_{2}$ due to illumination problems that could not be resolved in the available time (for more details see Vonlanthen (2010)). Because the two-dimensional data presented here were acquired with a three-dimensional set-up (cameras at $30^{\circ}$ to the normal in the $x_{1}-x_{3}$ plane) the data quality is slightly better for $u_{2}$ than for $u_{1}$.

For the main measurements in PEO solutions, the field of view was located in the vertical centre plane $x_{3}=0$ and centred at $x_{1}=30 M=48 \mathrm{~cm}, 30$ mesh widths $(M)$ 
(a)

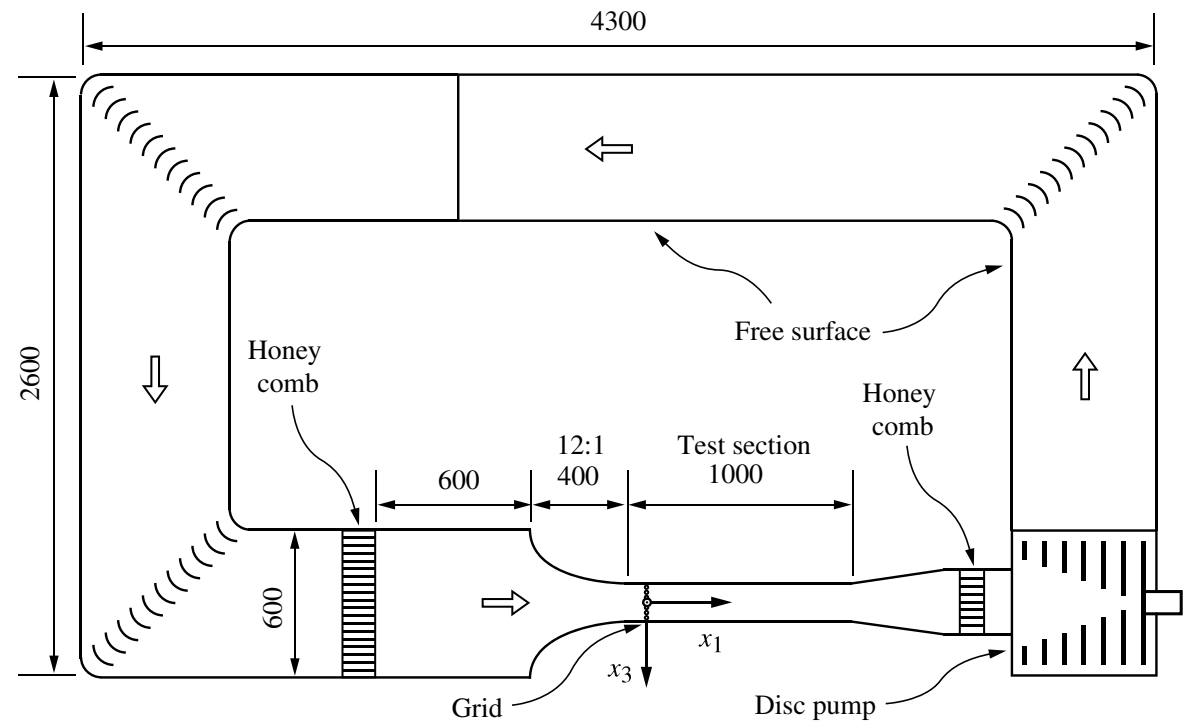

(b)

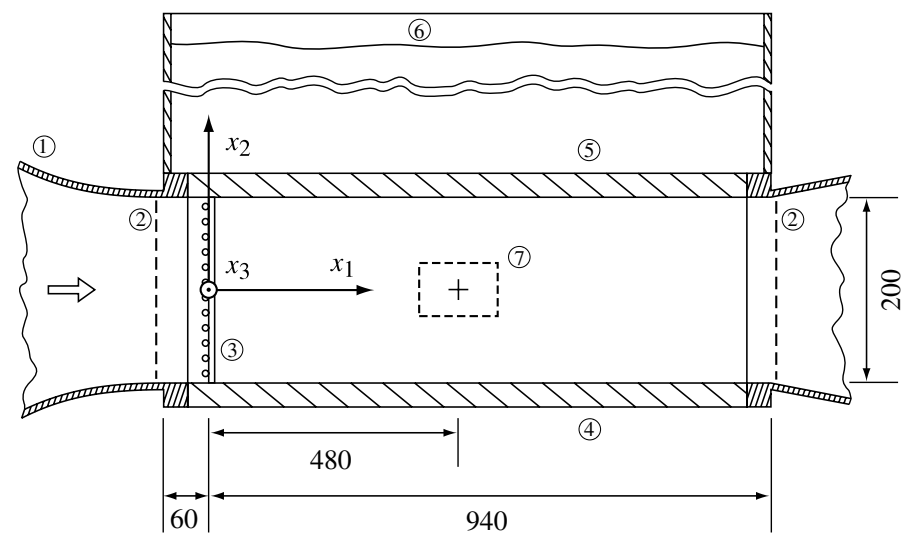

FIgURE 1. (a) Top view of the hydraulic tunnel (all dimensions in mm) with the 'Tesla' disc pump in the lower right corner downstream of the test section. (b) Side view of the $200 \mathrm{~mm} \times 150 \mathrm{~mm}$ test section (all dimensions in $\mathrm{mm}$ ); (1), 12:1 contraction; (2), upstream and downstream sections with pressure taps; (3), turbulence grid; (4), bottom window; (5), removable top window; (6), 'chimney' with free surface for easy access; 7), PIV field of view.

downstream of the grid, and $x_{2}=0$. The choice of the downstream measurement location of $30 \mathrm{M}$ was found to be the best compromise between high turbulence intensity and sufficient distance from the grid. In particular, at this location the variation of the turbulent energy dissipation rate across the field of view was less than $30 \%$ for the pure solvent (see Vonlanthen \& Monkewitz 2011). The size of the field of view was $64 \times 41 \mathrm{~mm}^{2}$ with the long dimension in the mean streamwise direction. Note that the field of view did not cover the entire CCD due to the stereoscopic set-up. Calibration was carried out in situ with a $10 \times 10 \mathrm{~cm}$ two-plane target aligned with the laser sheet. A final calibration adjustment was made by requiring that the mean stream wise velocity $U_{1}$ be constant over the field of view (the increase of $U_{1}$ over $64 \mathrm{~mm}$ 
due to boundary layer growth on the test section walls was only $0.3 \%$, well within the error bar) and $U_{2}$ be zero.

The flow was seeded with $9 \mathrm{~g}$ of polyamide tracer particles (Dantec), with an average diameter of $d_{p}=5 \mu \mathrm{m}$ and a density of $\rho_{p}=1.03 \mathrm{~g} \mathrm{~cm}^{-3}$. To avoid clumping of the seeding in the polymer solutions, it was added to the moderately agitated fluid shortly before the measurements with a small amount of detergent to lower the surface tension. The ability of the seeding to follow the small-scale structures of the flow is characterized by the Stokes number $d_{p}^{2} /\left(12 \beta \eta_{0}^{2}\right) \approx 2 \times 10^{-4}$, with the solvent-particle density ratio $\beta \equiv 3 \rho_{s} /\left(\rho_{s}+2 \rho_{p}\right) \approx 1$ and $\rho_{s} \approx 1 \mathrm{~g} \mathrm{~cm}^{-1}$, and by the particle volume fraction which was roughly $2.5 \times 10^{-6}$. According to Elghobashi (1994), the present seeding and turbulence parameters are approaching the regime where the particles enhance the turbulence energy dissipation, but this effect is still negligible compared with other experimental uncertainties.

The optimum time delay between laser pulses was found to be $\sim 200 \mu \mathrm{s}$, resulting in a mean particle displacement of roughly 10 pixels in the $x_{1}$ direction with an uncertainty of $\sim 0.06$ pixels. These choices resulted in a root mean square (r.m.s.) turbulent particle displacement of $\sim 0.5$ pixels. Hence, the small-scale motions of the flow were affected by measurement noise. The method to recover small-scale turbulence data from these noisy data is now described.

\subsection{PIV data processing}

TSI's Insight 3G software was used to process the stereoscopic PIV captures. Although this code supports deformation of the interrogation window (IW) to optimize correlations, it does not provide spatial velocity derivatives. Therefore, additional errors are introduced due to the subsequent differentiation of the velocity field. A multipass algorithm starting with an IW size of $128 \times 128$ pixels $^{2}$ and finishing with $32 \times 32$ pixels $^{2}$ was used together with a $50 \%$ IW overlap. After each pass, a validation procedure was applied to the vector field and spurious vectors were replaced by interpolating between valid neighbours. The outlier detection was based on a normalized median filter, which is well-adapted to velocity fields with strong gradients (Westerweel \& Scarano 2005). Since the number of outliers was generally below $1 \%$ of all vectors, their influence on the power spectra was negligible, as shown by Poelma, Westerweel \& Ooms (2006).

For each experiment, a total of 500 velocity fields were acquired with a sampling rate of $0.25 \mathrm{~Hz}$, owing to the very large CCD array. Considering that the integral time scale was of the order of $10 \mathrm{~ms}$, the recorded samples were statistically independent. After subtracting time averages from the velocity fields, the r.m.s. of $u_{1}^{\text {raw }}$ and $u_{2}^{\text {raw }}$ were determined, where the superscript ' $r a w$ ' indicates measurements not corrected for the effect of PIV windowing. Corresponding longitudinal and transverse power spectra along $x_{1}, E_{11}^{\text {raw }}\left(\kappa_{1}\right)$ and $E_{22}^{\text {raw }}\left(\kappa_{1}\right)$ were computed with a standard fast Fourier transform (FFT). Owing to the homogeneity of the turbulence in the $x_{2}$ direction, spectra could be averaged within each sample over lines $x_{2}=$ constant which were separated by two integral length scales or more, i.e. which were statistically independent.

To improve convergence, the spectra were further averaged over several velocity fields. Since the properties of PEO solutions changed during the experiment due to rupture of polymer chains, separate averages were taken over consecutive periods of $200 \mathrm{~s}$ corresponding to 50 velocity fields. During each of the $200 \mathrm{~s}$ periods, the fluid completed about two laps around the hydraulic tunnel, i.e. passed twice through grid and pump. This averaging time proved to be the best compromise between the requirements of stable fluid properties and convergence of spectral estimates: for the 
chosen averaging period, the $95 \%$ confidence interval still converged to within $20 \%$ of the averaged spectra.

A systematic attenuation arises from the spatial and the temporal sampling of the PIV technique, similar to the filtering effect of finite length hot wires identified by Wyngaard (1968). Following Lavoie et al. (2007), the filter function $\chi$ for an IW of width $w_{1}$, height $w_{2}$ and depth $w_{3}$ is

$$
\chi(\kappa)=\operatorname{sinc}\left(\frac{1}{2} \kappa_{1} U_{1} \Delta t\right) \prod_{i=1}^{3} \xi_{i} \quad \text { with } \xi_{i} \equiv \operatorname{sinc}\left(\frac{1}{2} \kappa_{i} w_{i}\right),
$$

where $\kappa_{i}$ is the wavenumber in the $i$ th direction, $\Delta t$ the time delay between consecutive frames of a PIV image pair, $U_{1}$ the local mean flow in the $x_{1}$ direction $\left(U_{2}\right.$ is negligible near the centreline) and $\operatorname{sinc}(z) \equiv \sin (z) / z$ the cardinal sine function. For all of the data presented in this paper, the time delay $\Delta t$ was $180 \mu \mathrm{s}$ for the $50 \mathrm{wppm}$ PEO solution and $160 \mu \mathrm{s}$ for the 25 and $100 \mathrm{wppm}$ solutions. The IW dimensions for all of the runs with PEO solutions were $\left(w_{1}, w_{2}, w_{3}\right)=(0.671 \mathrm{~mm}, 0.671 \mathrm{~mm}, 0.5 \mathrm{~mm})$.

As the PIV measurements were relatively noisy, the high wavenumber end of the spectra was significantly affected. According to Foucaut, Carlier \& Stanislas (2004), for streamwise power spectra $E_{i i}^{\text {raw }}\left(\kappa_{1}\right)$ the noise consists of a white noise $\mathscr{N}_{i i}^{(1)}$ modulated by the spectral function $\xi_{1}^{2}$ defined in (2.1). An additional unfiltered lowlevel white noise $\mathscr{N}_{i i}^{(2)}$ was found to produce a smoother steepening of spectra in the viscous dissipation range without affecting the lower wavenumbers. The uncorrected one-dimensional spectra are therefore related to the true velocity spectrum tensor $\Phi_{i j}(\boldsymbol{\kappa})$ and the wavenumber vector $\boldsymbol{\kappa}$ by

$$
E_{i i}^{r a w}\left(\kappa_{1}\right)=E_{i i}^{f i l}+\xi_{1}^{2} \mathscr{N}_{i i}^{(1)}+\mathscr{N}_{i i}^{(2)}
$$

with

$$
E_{i i}^{f i l}=2 \iint_{-\infty}^{\infty} \Phi_{i i}(\boldsymbol{\kappa}) \chi^{2}(\boldsymbol{\kappa}) \mathrm{d} \kappa_{2} \mathrm{~d} \kappa_{3} .
$$

The true one-dimensional spectra, on the other hand, are related to $\Phi_{i j}(\kappa)$ by

$$
E_{i i}\left(\kappa_{1}\right)=2 \iint_{-\infty}^{\infty} \Phi_{i i}(\kappa) \mathrm{d} \kappa_{2} \mathrm{~d} \kappa_{3} .
$$

The implicit problem (2.2)-(2.4) for $E_{i i}\left(\kappa_{1}\right)$ is solved approximately by using the spectral tensor for isotropic turbulence

$$
\Phi_{i j}(\kappa)=\frac{E(\kappa)}{4 \pi \kappa^{4}}\left(\kappa^{2} \delta_{i j}-\kappa_{i} \kappa_{j}\right),
$$

and replacing the true three-dimensional spectrum $E(\kappa)$, which depends only on the magnitude of $\kappa$, i.e. $\kappa \equiv \sqrt{\kappa_{i} \kappa_{i}}$, by a fitted model spectrum $E^{M}$ with the superscript ' $M$ ' standing for model. Denoting the modelled integrals (2.3) and (2.4) by $E_{i i}^{M-f i l}$ and $E_{i i}^{M}$, respectively, the correction scheme reduces to

$$
E_{i i}\left(\kappa_{1}\right)=\frac{E_{i i}^{M}\left(\kappa_{1}\right)\left[E_{i i}^{r a w}\left(\kappa_{1}\right)-\xi_{1}^{2} \mathscr{N}_{i i}^{(1)}-\mathscr{N}_{i i}^{(2)}\right]}{E_{i i}^{M-f i l}\left(\kappa_{1}\right)}=\frac{E_{i i}^{M}\left(\kappa_{1}\right) E_{i i}^{M-f i l}\left(\kappa_{1}\right)}{E_{i i}^{r a w}\left(\kappa_{1}\right)-\xi_{1}^{2} \mathscr{N}_{i i}^{(1)}-\mathscr{N}_{i i}^{(2)}} .
$$

The model spectrum $E^{M}$, a generalization of the model spectrum given by Pope (2008, $§ 6.5 .3$ ), will be developed in $\S 4$ (equation (4.9)). Suffice to say here that it has 


$$
\begin{array}{lcccccc}
v_{s}^{a} & \begin{array}{l}
U_{0}^{a} \\
\left(\mathrm{~cm}^{2} \mathrm{~s}^{-1}\right)
\end{array} & \begin{array}{c}
\Lambda_{1}^{a} \\
\left(\mathrm{~cm} \mathrm{~s}^{-1}\right)
\end{array} & \begin{array}{c}
\lambda^{a} \\
(\mathrm{~cm})
\end{array} & \begin{array}{c}
R e_{\lambda}^{a} \\
0.00941
\end{array} & \begin{array}{c}
\epsilon_{0, \text { decay }}^{a} \\
\left(\mathrm{~cm}^{2} \mathrm{~s}^{-3}\right)
\end{array} & \begin{array}{c}
\epsilon_{0, P I V} \\
\left(\mathrm{~cm}^{2} \mathrm{~s}^{-3}\right)
\end{array} \\
0.00941 & 120 \pm 5 & 1.3 & 0.17 & 100 & 152 & 145
\end{array}
$$

TABLE 1. Summary of turbulence parameters at $x_{1} / M=30$ in pure water: viscosity $v_{s}$, bulk flow velocity $U_{0}$, streamwise integral length scale $\Lambda_{1}$, Taylor microscale $\lambda$, corresponding turbulence Reynolds number $R e_{\lambda}$, dissipation rates $\epsilon_{0 \text {,decay }}$ from streamwise turbulence decay and $\epsilon_{0, P I V}$ directly from PIV. ( ${ }^{a}$ From Vonlanthen \& Monkewitz 2011.)

the same general structure of an inertial/elastic spectrum multiplied by $\mathscr{F}_{\Lambda}^{M}$ defining the spectral shape at the integral scale, and by a viscous cutoff function $\mathscr{F}_{\eta}^{M}$. The correction steps for $E_{i i}\left(\kappa_{1}\right)$ are summarized as follows.

(i) First, the white noise level $\mathscr{N}_{i i}^{(1)}$ is estimated directly from the measured turbulence spectra according to Foucaut et al. (2004). Since the noise contribution to the raw spectrum was in all cases much larger than the turbulence contribution at the wavenumber $\kappa_{1}^{-3 d b}=2.8 / w_{1}$ where the filter function $\xi_{1}^{2}\left(\kappa_{1}^{-3 d b}\right)=1 / 2$, one can estimate $\mathscr{N}_{i i}^{(1)} \approx 2 E_{i i}^{r a w}\left(\kappa_{1}^{-3 d b}\right)$. In practice, the factor 2 had to be adapted to equalize the two right-hand sides of (2.6). For the present spectra, this factor varied between 2.16 and 2.2 for $\mathscr{N}_{11}^{(1)}$ and 1.84 and 1.9 for $\mathscr{N}_{22}^{(1)}$, confirming that $u_{2}$ is of somewhat better quality than $u_{1}$. The additional unfiltered white noise $\mathscr{N}_{i i}^{(2)}$ never exceeded $10 \%$ of $\mathscr{N}_{11}^{(1)}$.

(ii) A first approximation of the model spectrum $E^{M}$ in (2.5) is obtained by matching the associated $E_{i i}^{M}(2.4)$ to the measured $E_{i i}^{f i l}=E_{i i}^{r a w}\left(\kappa_{1}\right)-\xi_{1}^{2} \mathscr{N}_{i i}^{(1)}-\mathscr{N}_{i i}^{(2)}$. For this, the dissipation rate $\epsilon_{0}$ in the Kolmogorov inertial range and the Lumley scale $\kappa_{L}$ (cf. $\S \S 4$ and 6) are chosen to match the two spectra beyond the integral scale. The low- $\kappa$ part of $E^{M}$ which is governed by $\mathscr{F}_{\Lambda}^{M}$ is then adjusted to fit $E_{i i}^{\text {raw }}\left(\kappa_{1}\right)-\xi_{1}^{2} \mathscr{N}_{i i}^{(1)}-\mathscr{N}_{i i}^{(2)}$ under the additional constraint of matching as closely as possible the variances $\left\langle u_{i}^{2}\right\rangle$ determined directly from the velocity fields (in most cases within $10 \%$ ). Owing to the large-scale non-isotropy of our grid turbulence, it was necessary to determine individual functions $\mathscr{F}_{\Lambda, i}^{M}$ to fit key features of the measured spectra at low $\kappa_{1}$, such as the crossing point of $E_{11}$ and $E_{22}$, and the variance of both velocity components.

(iii) In a last step, all of the parameters of $E^{M}$ affecting the high- $\kappa$ end of the spectra are fine-tuned such as to obtain the best match between $E_{i i}^{\text {raw }}\left(\kappa_{1}\right)-\xi_{1}^{2} \mathscr{N}_{i i}^{(1)}-\mathscr{N}_{i i}^{(2)}$ and $E_{i i}^{M-f i l}$ defined by (2.3).

The correction scheme (2.1)-(2.6) is illustrated in figure 5 of $\S 4$ for one spectrum in the pure solvent and one polymer spectrum, demonstrating that the noise subtraction is by far the most important correction step. The principal fitting parameters for the spectra of $\S 5$ are given in table 3 .

The complete PIV processing has been validated in water against the data gathered by laser Doppler and hot-film anemometry reported in Vonlanthen (2010) and Vonlanthen \& Monkewitz (2011). For comparison with PEO turbulence, the key parameters of our grid turbulence in pure water are compiled in table 1. 


\section{Characterization of PEO solutions}

The working fluids for the present study were low-concentration aqueous solutions of PEO WSR-301 supplied by Dow. Here, we concentrate on the main properties which are relevant for the interpretation of the experiments, namely the "viscosity averaged-molecular weight' $M_{\mu}$ and the time constant $\tau$ on which stretched PEO molecules contract into a 'ball'. For the detailed rheometry, the reader is referred to Vonlanthen (2010).

The viscosity-averaged or effective molecular weight $M_{\mu}$ is obtained from the intrinsic viscosity $[\mu]$ via the Mark-Houwink relation with the parameters determined by Tirtaatmadja, McKinley \& Cooper-White (2006) for aqueous Polyox solutions with $8 \times 10^{3} \leqslant M_{\mu} \leqslant 5 \times 10^{6} \mathrm{~g} \mathrm{~mol}^{-1}$ :

$$
[\mu]=K\left(M_{\mu} / M_{u}\right)^{3 \beta-1}=0.072\left(M_{\mu} / M_{u}\right)^{0.65},
$$

where the prefactor $K$ is given in units of $\mathrm{cm}^{3} \mathrm{~g}^{-1}$ and $M_{u}=1 \mathrm{~g} \mathrm{~mol}^{-1}$ is the molar mass constant. The exponent $3 \beta-1=0.65$ yields a solvent quality parameter of $\beta=0.55$, indicating that water is a relatively good solvent of PEO. The limiting values of $\beta$ are 0.5 for a theta solvent and 0.6 for a good solvent, corresponding to weak and strong polymer-solvent interaction, respectively.

The zero-shear intrinsic viscosity $[\mu]$ characterizes the contribution of the polymer to the solution viscosity as the polymer concentration $c$ approaches zero. It is defined as (see e.g. Morrison 2001)

$$
[\mu]=\lim _{c \rightarrow 0} \mu_{\text {red }}=\lim _{c \rightarrow 0} \mu_{\text {inh }}
$$

with

$$
\mu_{\text {red }}=\frac{\mu-\mu_{s}}{c \mu_{s}} \text { and } \quad \mu_{\text {inh }}=\frac{\ln \left(\mu / \mu_{s}\right)}{c},
$$

where $\mu_{s}$ is the solvent viscosity. The PEO concentration $c$ will be specified throughout the paper in wppm, i.e. weight of PEO per weight of solvent, but note that dimensional homogeneity of the equations containing $c$ requires $c$ to be in units of $\mathrm{g} \mathrm{cm}^{-3}$. For water as a solvent the conversion is with sufficient accuracy $1 \mathrm{wppm}=10^{-6} \mathrm{~g} \mathrm{~cm}^{-3}$. To determine $[\mu]$, the viscosity of solutions with PEO concentrations between 20 and $500 \mathrm{wppm}$ was measured with an Ubbelohde capillary viscometer (solutions with more than 500 wppm were strongly affected by shear thinning). The viscometer was placed in a water bath maintained at constant temperature of $24 \pm 0.1{ }^{\circ} \mathrm{C}$. Each measurement was repeated nine times to attain an estimated uncertainty of $2 \%$ (viscosities determined for distilled water were within $0.5 \%$ of published values). The resulting $\mu_{\text {red }}$ and $\mu_{\text {inh }}$ are shown in figure 2 .

Despite the large error bars obtained by standard error propagation, the data in figure 2 are very consistent and show a distinctly different behaviour below and above 100 wppm. A similar behaviour has been reported by Sylvester \& Tyler (1970) who speculated that the sudden change is due to polymer entanglements which start to form around $100 \mathrm{wppm}$. This is also consistent with the clear break in the characteristics of an impinging turbulent jet between 50 and 100 wppm observed by Mejia-Alvarez \& Christensen (2011). We adhere to the view that the change of properties between 50 and 100 wppm corresponds to the transition between truly dilute solutions where there is essentially no interaction between PEO molecules and a regime where polymer entanglement is significant even though the critical coil overlap concentration $c^{*}$ is $0.77 /[\mu] \approx 500$ wppm, according to standard wisdom (see, e.g., Graessley 1980). The 


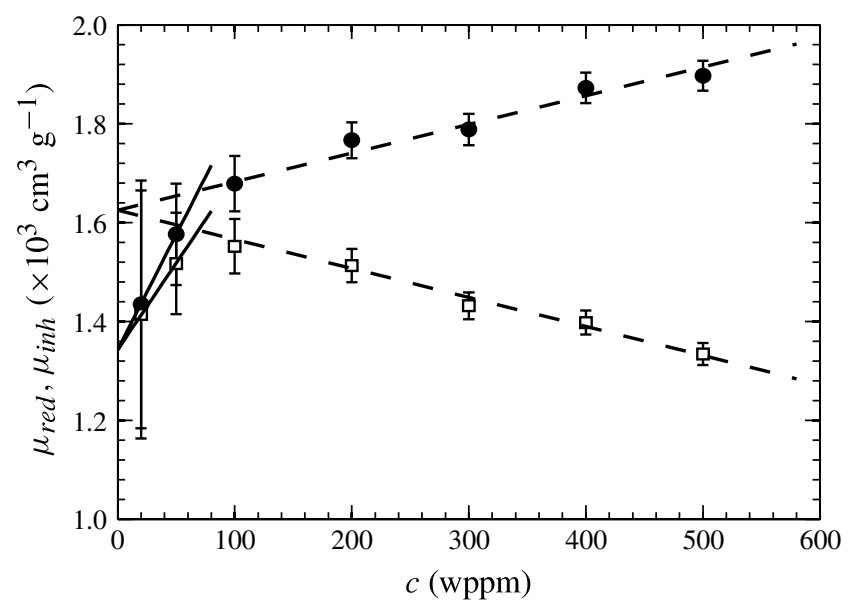

FIGURE 2. Linear extrapolations of the reduced ( $)$ and the inherent viscosity ( $\square$ ) to find the two intrinsic viscosities for $c \leqslant 50 \mathrm{wppm}(-)$ and $c \geqslant 100 \mathrm{wppm}(--)$.

change in fluid properties between 50 and $100 \mathrm{wppm}$ is also reflected in a qualitative change of pressure drop across the grid (see figure 5.1 in Vonlanthen 2010).

The two extrapolations in figure 2 yield the intrinsic viscosities $[\mu](c \leqslant 50 \mathrm{wppm})=$ $1360 \pm 350 \mathrm{~cm}^{3} \mathrm{~g}^{-1}$ and $[\mu](c \geqslant 100 \mathrm{wppm})=1630 \pm 55 \mathrm{~cm}^{3} \mathrm{~g}^{-1}$. These values are in line with published values (see, e.g., Kalashnikov \& Vlasov 1978; Tirtaatmadja et al. 2006), although $1360 \mathrm{~cm}^{3} \mathrm{~g}^{-1}$ is at the lower end of the spectrum. Using the Mark-Houwink relation (3.1) the measured [ $\mu]$ yield $M_{\mu}$ of $3.8 \times 10^{6}$ and $5 \times 10^{6} \mathrm{~g} \mathrm{~mol}^{-1}$, respectively. Both values are well within the distribution of molecular weights in Polyox WSR-301 reported for instance by Morgan \& Pike (1972).

The other quantity relevant to the interaction of polymer molecules with turbulence is the time constant $\tau$ for the contraction of stretched molecules into a ball. Widely different models exist to estimate $\tau$ and only the Maxwell model for a continuum, the Rouse-Zimm molecular model and the Rozhkov correlation (Rozhkov, Prunet-Foch \& Vignes-Adler 2003) are mentioned here.

The $N$-mode Maxwell model (see, e.g., Ferry 1980) yields

$$
\tau_{M}=\left(\mu_{0}-\mu_{s}\right) \frac{M_{\mu}}{c R T} \sum_{j=1}^{N} \hat{\tau}_{j}
$$

with

$$
\mu_{0} / \mu_{s}=1+1.05(c[\mu])+0.46(c[\mu])^{2}+0.24(c[\mu])^{3},
$$

where $\hat{\tau}_{j}$ is the rescaled non-dimensional relaxation time of the $j$ th dashpot-spring element, $\mu_{s}=0.00911 \mathrm{~g} \mathrm{~cm}^{-1} \mathrm{~s}^{-1}$ at $T=297 \mathrm{~K}$ and $R=8.3145 \times 10^{7} \mathrm{erg} \mathrm{mol}^{-1} \mathrm{~K}^{-1}$ the universal gas constant. The solution viscosity at zero shear, $\mu_{0}$, was fitted to the viscometer and rotational rheometer measurements (Bohlin C-VOR 150 with doublegap sample holder) using $[\mu]=1630 \mathrm{~cm}^{3} \mathrm{~g}^{-1}$.

The mode relaxation times were deduced from the storage and loss moduli obtained with oscillatory shear experiments in the rotational rheometer with a cone-and-plate set-up. Owing to the limited sensitivity of the rheometer, the storage and loss moduli could only be determined for concentrated solutions of 3000 and $5000 \mathrm{wppm}$ and 


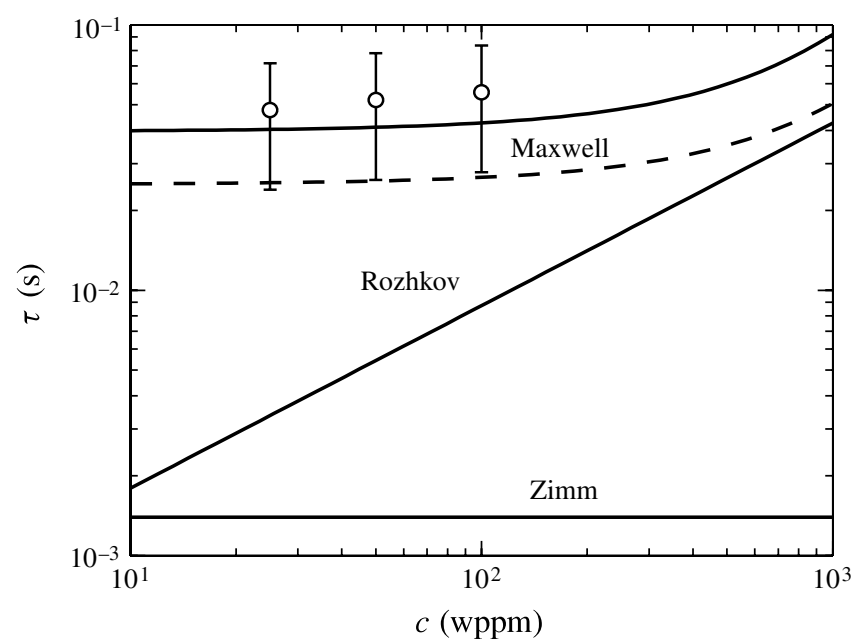

FIgURE 3. Polymer relaxation times $\tau$ : Maxwell for $M_{\mu}=3.8 \times 10^{6} \mathrm{~g} \mathrm{~mol}^{-1}(--)$ and $M_{\mu}=5 \times 10^{6} \mathrm{~g} \mathrm{~mol}^{-1}$ (一); Rouse-Zimm (equation (3.4)) for $M_{\mu}=5 \times 10^{6} \mathrm{~g} \mathrm{~mol}^{-1}$; Rozhkov (equation (3.5)) for $[\mu]=1630 \mathrm{~cm}^{3} \mathrm{~g}^{-1} ; \bigcirc, \tau_{L}$ deduced from the fitted initial Lumley lengths (see $\S 6$ ), including $50 \%$ error bars.

the model had to be limited to two modes $(N=2)$. The resulting $\left(\hat{\tau}_{1}+\hat{\tau}_{2}\right)=12.3$ yielded the surprisingly reasonable $\tau_{M}$ shown in figure 3 , considering the extrapolation to 100 wppm and below. For more detail, see Vonlanthen (2010).

The Rouse-Zimm model (Rodd et al. 2007) can, similarly to the Maxwell model, be expressed in terms of bulk properties as

$$
\tau_{Z}=0.47 \frac{\mu_{s}[\mu] M_{\mu}}{R T}=0.0338 \frac{\mu_{s} M_{u}}{R T}\left(\frac{M_{\mu}}{M_{u}}\right)^{1.65},
$$

where the prefactor has been evaluated for the solvent quality parameter of $\beta=0.55$ and (3.1) has been used to find the second expression. Finally, the Rozhkov correlation for the relaxation time $\tau_{R}$ (Rozhkov et al. 2003) is

$$
\tau_{R}=0.0305(c[\mu])^{0.688},
$$

where the prefactor corresponds to $[\mu]=1630 \mathrm{~cm}^{3} \mathrm{~g}^{-1}$.

All three relaxation times are shown in figure 3 for the $M_{\mu}$ determined from figure 2. As already mentioned, in the range 25-100 wppm only the Maxwell model based on the rheometry of our PEO solutions yields relaxation times of the order of the times deduced from the Lumley scales of $\S 6$. The difference between $\tau_{L}$ or $\tau_{M}$ and $\tau_{R}$ for the concentrations used in the present experiments is likely due to the strongly nonlinear uncoiling of polymer chains (see, e.g., Hinch 1994) and the difference between the flows in which the relaxation times were identified, i.e. turbulence, rotational rheometer and steady laminar extensional flow. Finally, $\tau_{Z}$ of the Rouse-Zimm model is similar to $\tau_{R}$ for truly dilute solutions, but does not take into account the effect of increasing concentration.

The relevant fluid properties for the three PEO concentrations considered in this investigation are given in table 2 . 


\begin{tabular}{|c|c|c|c|c|c|c|}
\hline $\begin{array}{c}c \\
\text { wppm) }\end{array}$ & $\begin{array}{c}m_{P E O} \\
(\mathrm{~g})\end{array}$ & $\begin{array}{c}T \\
\left({ }^{\circ} \mathrm{C}\right)\end{array}$ & $\begin{array}{c}v_{s} \\
\left(\mathrm{~cm}^{2} \mathrm{~s}^{-1}\right)\end{array}$ & $\begin{array}{c}v \\
\left(\mathrm{~cm}^{2} \mathrm{~s}^{-1}\right)\end{array}$ & $\begin{array}{c}{[\mu]} \\
\left(\mathrm{cm}^{3} \mathrm{~g}^{-1}\right)\end{array}$ & $\begin{array}{c}M_{\mu} \\
\left(\mathrm{g} \mathrm{mol}^{-1}\right)\end{array}$ \\
\hline 25 & 18 & 22.2 & 0.0095 & 0.0099 & 1360 & $10^{6}$ \\
\hline 50 & 36 & 1 & 0.0098 & & 13 & $3.8 \times 10^{6}$ \\
\hline 100 & 720 & 22.2 & 0.0095 & 0.0107 & 1630 & $5.0 \times 10^{6}$ \\
\hline
\end{tabular}

TABLE 2. Summary of fluid properties for the three PEO concentrations investigated: $m_{P E O}$, mass of dry PEO powder; $v_{s}$ and $v$, kinematic viscosities of the pure solvent and the solution, respectively; $[\mu]$, zero-shear intrinsic viscosity; $M_{\mu}$, viscosity-averaged molecular weight.

\section{Model spectrum}

Owing to the limited scaling ranges of our experimental spectra, it is not possible to reliably fit power laws. Therefore, it is imperative to develop a complete model spectrum that can be fitted to the data as a whole.

As is evident from the spectra in the next $\S 5$, there is a clear break away from the Kolmogorov inertial $-5 / 3$ range, which is not affected by the polymers, into an 'elastic' subrange. This new spectral region is separated from the inertial cascade by the Lumley scale $\ell_{L} \equiv 1 / \kappa_{L}$ (in analogy to the definition of the Kolmogorov length $\eta_{0}$, the factor $2 \pi$ is omitted in the definition of $\ell_{L}$ ), determined by the elastic properties of the fluid and the turbulence dissipation rate (cf. $\S 6$ where the time dependence of $\ell_{L}$ is related to polymer degradation). In the elastic subrange, the turbulence kinetic energy cascading down from the inertial subrange is partly converted into elastic energy of the extended polymer coils and partly dissipated as solvent friction required to extend the polymer chains. As the polymer chains coil up again, a part of the stored elastic energy is also dissipated due to viscous drag opposing the contraction and to interactions between monomers of a single polymer (Ouellette et al. 2009). The residual part of the elastic energy is transformed back to turbulent kinetic energy (this has been termed 'back reaction' by Balkovsky et al. 2001) and passed on to smaller eddies. The process, henceforth called 'elastic dissipation', continues until either viscous dissipation takes over or the turbulent 'eddies' become smaller than the stretched polymer molecules, a case that requires a much higher Reynolds number than in the present experiments.

Hinch (1977) was to the best of the authors' knowledge the first to speculate that the bulk effect of polymer chains very much shorter than the flow scales was to maintain the local instantaneous rate of strain below a critical rate of strain, directly related to the time constant of the polymer (see $\S 6$ ). From the estimate of the rate of strain $\left(E \kappa^{3}\right)^{1 / 2}$ in terms of energy spectral density $E(\kappa)$ and wavenumber $\kappa$ it follows immediately that a constant rate of strain in the elastic subrange corresponds to $E \propto \kappa^{-3}$ seen for instance by Warholic et al. (1999) and in the numerical study of Berti et al. (2006). It also implies that the energy cascade down this -3 slope is self-regulating: if the energy falls below, the rate of strain becomes subcritical and the polymer inactive. Hence, the decay reverts to the $-5 / 3$ slope until the critical strain rate is again attained. If the energy deviates above the critical $\kappa^{-3}$ decay, the polymers 'work harder' to dissipate the excess energy, provided of course their concentration is sufficient. Fouxon \& Lebedev (2003), on the other hand, argued for an energy decay steeper than $\kappa^{-3}$ based on the convergence of certain integrals in the limit of infinite Reynolds number. However, their argument may not be conclusive as the 
physics changes when flow scales become smaller than the polymer chain length. Our data support a scaling $E(\kappa) \propto \kappa^{-3}$, but the limited scaling ranges do not allow to argue about small differences in the scaling exponent. Therefore, the exponents of the inertial and elastic subranges are fixed in the following to $\kappa^{-5 / 3}$ and $\kappa^{-3}$, respectively.

The energy flux $\epsilon$ corresponding to a spectrum $E \propto \kappa^{-3}$ beyond the Lumley wavenumber $\kappa_{L}$ is

$$
\epsilon(\kappa) / \epsilon_{0}=\tilde{\kappa}^{-2} \text { for } \tilde{\kappa} \equiv \kappa / \kappa_{L} \geqslant 1,
$$

where $\epsilon_{0}$ is the constant energy flux in the Kolmogorov inertial range $\kappa \leqslant \kappa_{L}$. In the 'strong back reaction regime' (Balkovsky et al. 2001) the Lumley wavenumber depends on $M_{\mu}, \epsilon_{0}$ and the polymer type, but not on concentration. Relation (4.1) corresponds to an elastic dissipation at the scale $\kappa$ of $\mathrm{d} \epsilon / \mathrm{d} \kappa=-2\left(\epsilon_{0} / \kappa_{L}\right) \tilde{\kappa}^{-3}$. The fact that this elastic dissipation is proportional to the eddy volume for all $\kappa \geqslant \kappa_{L}$ suggests a generalized ansatz where the dissipation is reduced by the same factor $\Gamma$ at all scales in the case where the polymer concentration is insufficient to maintain the strain rate below the critical rate. Hence, we model the elastic dissipation as

$$
\frac{\mathrm{d} \epsilon}{\mathrm{d} \kappa}=-2 \Gamma\left(c ; M_{\mu} ; c / c^{*} ; \text { Polymer type }\right) \frac{\epsilon_{0}}{\kappa_{L}} \tilde{\kappa}^{-3},
$$

where $0 \leqslant \Gamma \leqslant 1$ depends on the concentration, molecular weight, degree of dilution and polymer type. For a given polymer type and low concentration $c / c^{*} \ll 1$, the strong back reaction regime is reached at a critical concentration $c_{c r i t}$ defined by $\Gamma\left(c_{\text {crit }} ; M_{\mu}\right)=1$.

Integrating (4.2) and matching to the Kolmogorov inertial range yields for $\tilde{\kappa} \geqslant 1$

$$
\epsilon(\kappa) / \epsilon_{0}=1-\Gamma+\Gamma \tilde{\kappa}^{-2}
$$

and

$$
E / E_{L}=\tilde{\kappa}^{-5 / 3}\left[1-\Gamma+\Gamma \tilde{\kappa}^{-2}\right]^{2 / 3},
$$

where $E_{L} \equiv \alpha \epsilon_{0}^{2 / 3} \kappa_{L}^{-5 / 3}$ is the energy spectral density at the Lumley scale and $\alpha=3 / 2$ the standard Kolmogorov constant.

From (4.3) the new viscous scale $\eta \equiv 1 / \kappa_{\eta}=v_{s}^{3 / 4} \epsilon^{-1 / 4}$ is obtained as usual by setting the turbulence Reynolds number equal to one. This results in a cubic equation for $\eta$ with the approximate solution

$$
\eta \cong \eta_{0}\left[1-\Gamma+\Gamma R e_{L}^{-1}\right]^{-1 / 4} \quad \text { with } R e_{L} \equiv \epsilon_{0}^{1 / 3} \kappa_{L}^{-4 / 3} v_{s}^{-1}=\left(\eta_{0} \kappa_{L}\right)^{-4 / 3}
$$

the characteristic turbulence Reynolds number at the Lumley scale and $\eta_{0} \equiv v_{s}^{3 / 4} \epsilon_{0}^{-1 / 4}$ the Kolmogorov scale without polymers. This approximate solution is exact in the limits $\Gamma=0$ and $\Gamma=1$, and deviates from the exact solution by less than $1 \%$ for $R e_{L}$ as low as 50. From (4.4) and (4.5) one obtains easily that

$$
\eta(\Gamma=1) / \eta_{0}=\operatorname{Re}_{L}^{1 / 4} \quad \text { and } \quad \frac{E\left(\kappa=\eta^{-1} ; \Gamma=1\right)}{E\left(\kappa=\eta_{0}^{-1} ; \Gamma=0\right)}=\operatorname{Re}_{L}^{-1 / 4}
$$

The energy spectrum $E(\kappa)$ (equation (4.4)) still needs to be complemented by a viscous cut-off function $\mathscr{F}_{\eta}$. Following the approximate approach of Corrsin, described by Tennekes \& Lumley $(1972, \S 8.4)$, the energy flux equation is augmented by the elastic flux to read

$$
\frac{\mathrm{d} \epsilon}{\mathrm{d} \kappa} \cong-2 \Gamma \frac{\epsilon_{0}}{\kappa_{L}} \tilde{\kappa}^{-3} \mathscr{F}_{\eta}-2 \nu_{s} \kappa^{2} E \mathscr{F}_{\eta},
$$


where $E$ is given by (4.4). Writing the energy flux $\epsilon=\alpha^{-3 / 2} \kappa E \mathscr{F}_{\eta} \sigma$ with the strain rate $\sigma=\kappa^{3 / 2} E^{1 / 2}$ (without $\mathscr{F}_{\eta}$ !) yields a linear first-order equation for $\mathscr{F}_{\eta}$ with the approximate solution

$$
\ln \mathscr{F}_{\eta}=-\frac{3}{2} \alpha\left(\kappa \eta_{0}\right)^{4 / 3} \frac{\left[1-\Gamma+\Gamma \tilde{\kappa}^{-2}\right]^{2 / 3}}{1-\Gamma+\frac{3}{2} \Gamma \tilde{\kappa}^{-2}} \text { for } \tilde{\kappa} \geqslant 1 .
$$

The result (4.8) is again exact (within the limitations of the approach) for $\Gamma=0$ and $\Gamma=1$ and a very good approximation in between. For the case of $\Gamma=0$, the viscous damping function $\mathscr{F}_{\eta}$ of (4.8) is known to produce a departure of $E$ from $\kappa^{-5 / 3}$ which is too gradual. The best fits to our spectra were obtained by replacing the 'solvent factor' $(3 / 2) \alpha\left(\kappa \eta_{0}\right)^{4 / 3}$ in (4.8) by Pope's expression (cf. $\S 6.5 .3$ of Pope 2008). Furthermore, to avoid having to use separate fits for $\kappa<\kappa_{L}$ and $\kappa \geqslant \kappa_{L}$, all of the terms $\Gamma \tilde{\kappa}^{-2}$ are modified to $\Gamma\left(1+\tilde{\kappa}^{\gamma}\right)^{-2 / \gamma}$, where $\gamma$ controls the rounding of the corner between the inertial and elastic parts of the spectrum $(\gamma \rightarrow \infty$ corresponds to a sharp corner; a constant $\gamma=20$ has proven adequate for all of the fits in this study). With the usual factor $\mathscr{F}_{\Lambda}$ describing the energy injection scales, the complete three-dimensional model spectrum $E^{M}$, with superscript ' $M$ ' for model, is finally

$$
E^{M}(\kappa)=\alpha \epsilon_{0}^{2 / 3} \kappa^{-5 / 3}\left[1-\Gamma+\Gamma\left(1+\tilde{\kappa}^{\gamma}\right)^{-2 / \gamma}\right]^{2 / 3} \mathscr{F}_{\Lambda}^{M}\left(\kappa / \kappa_{\max }\right) \mathscr{F}_{\eta}^{M}\left(\kappa \eta_{0} ; \tilde{\kappa}\right)
$$

with

$$
\mathscr{F}_{\Lambda}^{M}=\left\{\left(\kappa / \kappa_{\max }\right)\left[\left(\kappa / \kappa_{\max }\right)^{2 \beta}+\frac{5}{12}\right]^{-1 /(2 \beta)}\right\}^{17 / 3}
$$

and

$$
\ln \mathscr{F}_{\eta}^{M}=-\left\{5.2\left[\left(\kappa \eta_{0}\right)^{4}+0.4^{4}\right]^{1 / 4}-2.08\right\} \frac{\left[1-\Gamma+\Gamma\left(1+\tilde{\kappa}^{\gamma}\right)^{-2 / \gamma}\right]^{2 / 3}}{1-\Gamma+\frac{3}{2} \Gamma\left(1+\tilde{\kappa}^{\gamma}\right)^{-2 / \gamma}} .
$$

The above $\mathscr{F}_{\Lambda}^{M}$ is essentially Pope's expression (cf. $\S 6.5 .3$ of Pope 2008), rewritten in terms of $\kappa / \kappa_{\max }$, with $\kappa_{\max }$ the wavenumber where $E$ is maximum, and slightly generalized by the introduction of a variable exponent $\beta$. This provides more flexibility to take into account the (moderate) non-isotropy of large-scale energy injection by our grid (cf. Vonlanthen \& Monkewitz 2011). Note also that the Saffman form of the spectrum, i.e. $E(\kappa) \propto \kappa^{2}$ for $\kappa \rightarrow 0$, is used throughout this study as it has proven superior for all the fits. Specifically, it provided a better match of r.m.s. velocities after fitting the high- $\kappa$ end of the spectra.

Examples of the model spectra (4.9) for different $\Gamma$ are shown in figure 4 which reveals a most interesting feature of the model spectrum: for $0<\Gamma<1$ and sufficiently high Reynolds number, the spectrum, beyond a limited wavenumber range with elastic dissipation, reverts back to a second K41 inertial range at a lower $\epsilon_{1}<\epsilon_{0}$. Such spectra have yet to be seen experimentally, but resemble the spectra obtained by Benzi et al. (2003) (see also §7). The inset in figure 4 also shows the one-dimensional spectra $E_{i i}\left(\kappa_{1}\right)$ deduced from $E^{M}(\kappa ; \Gamma=1)$, together with a method to determine $\kappa_{L}$ directly from one-dimensional spectra. With a reasoning similar to the one relating the Kolmogorov coefficient $\alpha=3 / 2$ in the three-dimensional spectrum to the coefficients $\alpha_{1}=(18 / 55) \alpha$ and $\alpha_{2}=(24 / 55) \alpha$ of the one-dimensional longitudinal and transverse inertial spectra, the three-dimensional Lumley length $\ell_{L}$ for $\Gamma=1$ is found to be 


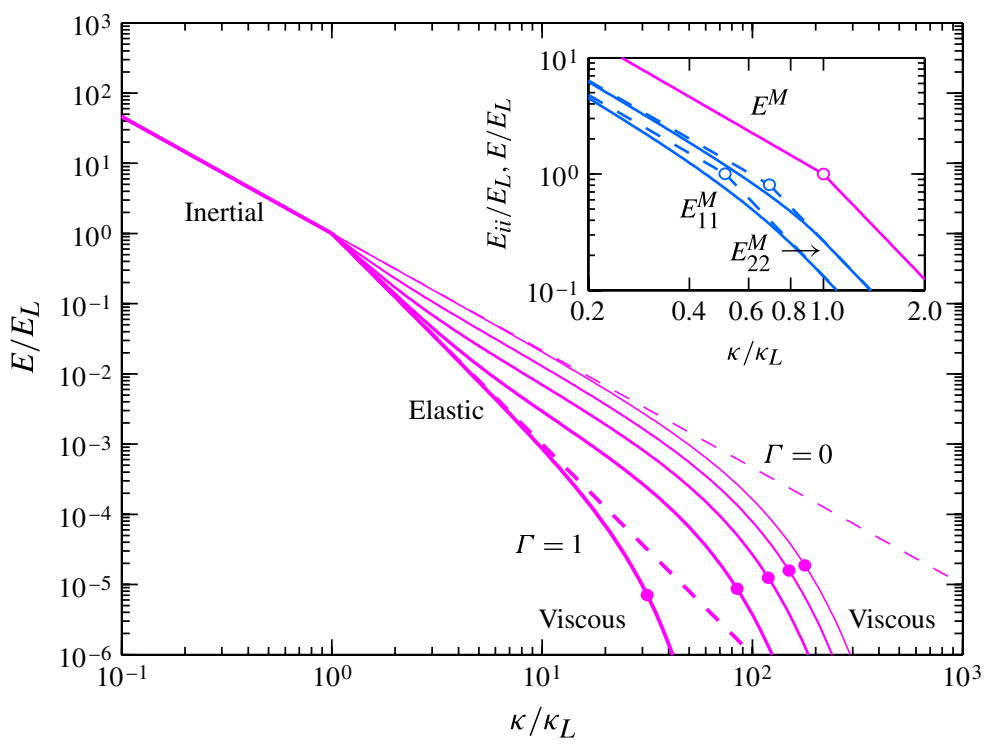

FIGURE 4. Model spectra (4.9) for $R e_{L}=10^{3}$, indicating the inertial, elastic and viscous ranges. The line width indicates the PEO concentration, ranging from $\Gamma=0$ (no PEO) with inertial range $E^{M} \propto \kappa^{-5 / 3}$, to $\Gamma=1$ (enough PEO to limit the strain rate) with elastic range $E \propto \kappa^{-3}$. Intermediate values are $\Gamma=0.95,0.8$ and 0.5 ; $\odot$, corresponding dissipation scales according to (4.5). Inset: close-up view of $E^{M}(\kappa ; \Gamma=1)$ at infinite Reynolds number with the associated normalized one-dimensional spectra $E_{11}$ and $E_{22} ; \bigcirc$, corresponding intersections $\kappa_{L, i}$ of the $-5 / 3$ and -3 power laws (equation (4.10)).

related to the intersections $\kappa_{L, i}$ between the $\kappa_{1}^{-5 / 3}$ and $\kappa_{1}^{-3}$ fits to $E_{i i}$ by

$$
\ell_{L}(\Gamma=1)=(11 / 27)^{3 / 4} \kappa_{L, 1}^{-1}=(11 / 18)^{3 / 4} \kappa_{L, 2}^{-1} .
$$

It is evident that this method to determine $\ell_{L}$ is reliable only if both the inertial and elastic scaling ranges are sufficiently long which is not the case in the present experiments. As seen in figures 6 and 7, at early times the inertial range is virtually non-existent and at late times the elastic range merges into the dissipation range.

\section{Experimental energy spectra}

Energy spectra in PEO solutions of three different concentrations, 25, 50 and 100 wppm, were acquired at $x_{1}=30 M=48 \mathrm{~cm}$ downstream of the grid. At the start of each experimental run the pump was accelerated quickly and the flow was allowed to stabilize during the first $100 \mathrm{~s}$ before the PIV recordings were started. As the approximate desired mean velocity was reached already after $10 \mathrm{~s}$, the fluid went around the facility about once during this start-up period of $100 \mathrm{~s}$. Since this first 'lap' (1 lap = $100 \mathrm{~s}$ ) already contributed to the degradation of polymers, it will be included in the lap count. As the polymers were degrading rather rapidly, the spectra were averaged over consecutive periods of only $200 \mathrm{~s}$, corresponding to two laps, which represented a compromise between the convergence of the spectra and the ability to follow their temporal evolution (see also $\$ 2.3$ ). The result is what we believe to be the first 'movie' of the spectral evolution of grid turbulence in PEO solutions. The present data (figures 6 and 7) lend strong support to a rather sharp 

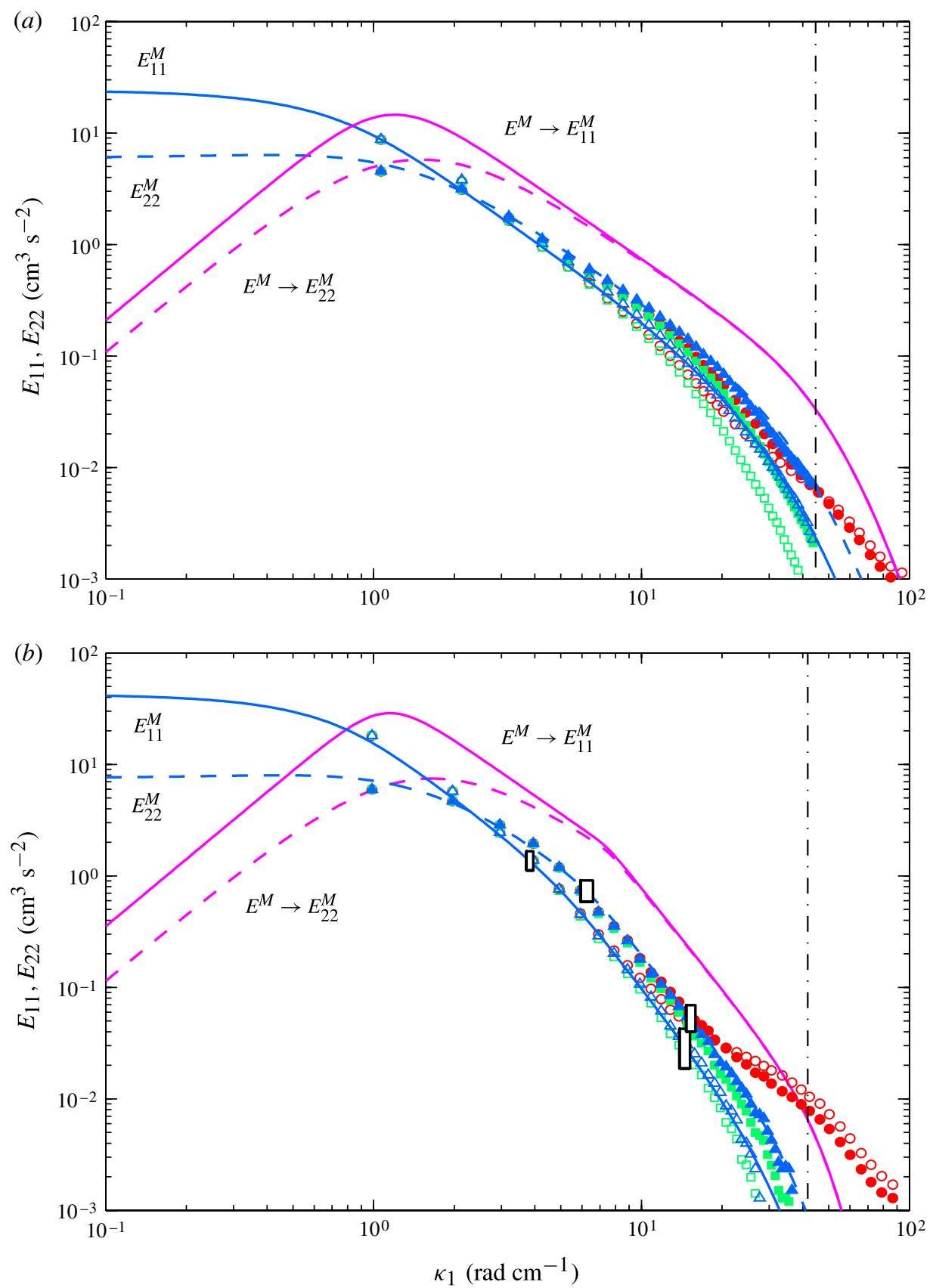

FIGURE 5. Examples of spectral correction steps for grid turbulence $(a)$ in plain water and $(b)$ in a 50 wppm PEO solution after 4 circuits in the tunnel (data acquired between 300 and $500 \mathrm{~s}$ after start). Solid and broken lines represent model spectra pertaining to streamwise and transverse velocity fluctuations, respectively: purple lines, fitted three-dimensional model spectra $E^{M}(\kappa)$ (4.9) with individualized low- $\kappa$ part and corresponding $E_{i i}^{M}\left(\kappa_{1}\right)$ (blue lines). The main fitting parameters are given in table 3 . Streamwise $(\bigcirc)$ and transverse $(\mathbf{O})$, raw experimental spectra $E_{i i}^{\text {raw }}\left(\kappa_{1}\right) ; \square, \mathbf{\square}$, raw spectra minus noise $E_{i i}^{\text {raw }}\left(\kappa_{1}\right)-\xi_{1}^{2} \mathscr{N}_{i i}^{(1)}-\mathscr{N}_{i i}^{(2)}$; $\triangle, \boldsymbol{\Delta}$, fully corrected spectra according to $(2.6) ;-\cdot-$, cut-off wavenumber $\kappa_{1}^{-3 d b} ; \square$, error estimates near the Lumley and viscous scales (see the text for details). 


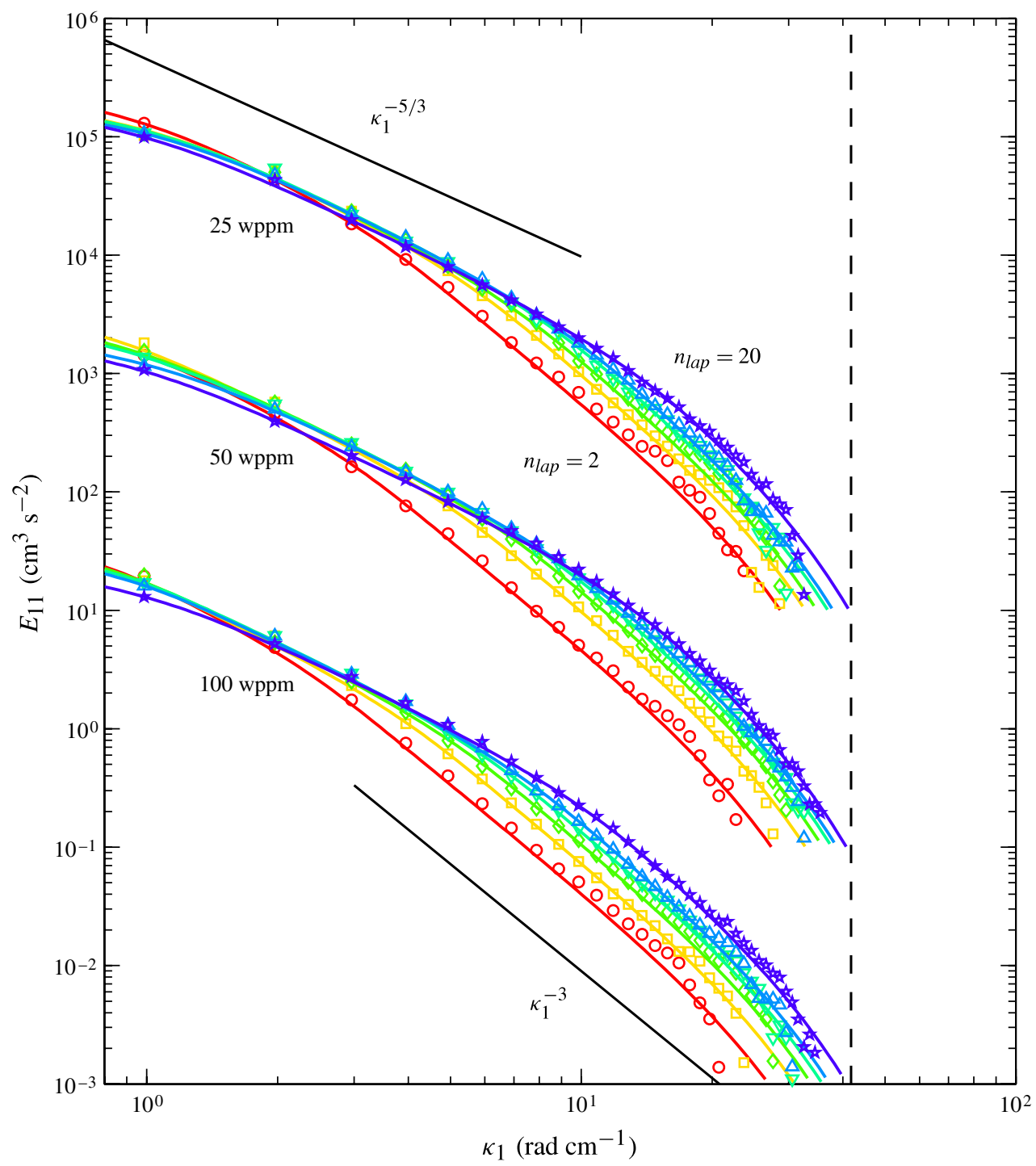

FIGURE 6. Fully corrected streamwise velocity spectra (equation (2.6)) at $x_{1} / M=30$ in PEO solutions after: $\bigcirc, 2 ; \square, 4 ; \diamond, 6 ; \nabla, 8 ; \Delta, 10 ; \downarrow, 20$ circuits in the tunnel; - , fitted model spectra $E_{i i}^{M}\left(\kappa_{1}\right) ;--$, cut-off wavenumber $\kappa_{1}^{-3 d b}$. The spectra in 50 and 25 wppm solutions are shifted up by $10^{2}$ and $10^{4}$, respectively. Experimental parameters are given in tables 2 and 3 .

transition in the three-dimensional spectrum from a Kolmogorov inertial range to an energy decay $E(\kappa) \propto \kappa^{-3}$. Needless to say that long-time averaging of the spectra would have completely washed out this sharp transition. It is useful to recall here that a sharp corner in the three-dimensional spectra translates into rounded transitions in the one-dimensional spectra, as seen in the inset of figures 4 and $5(b)$.

Before showing the corrected experimental spectra, it is useful to visualize in figure 5 an example of the detailed correction procedure described in $\S 2.3$. The figure demonstrates that: 


\begin{tabular}{lcccccccccc}
\hline $\begin{array}{l}\text { PEO } \\
\text { conc. } \\
\text { (wppm) }\end{array}$ & $\begin{array}{c}\text { Time } \\
\text { after start } \\
(\text { laps })\end{array}$ & $U_{0}$ & $\epsilon_{0}$ & $\eta$ & $\kappa_{L}$ & $\kappa_{\max , 1}$ & $\kappa_{\max , 2}$ & $\beta_{1}$ & $\beta_{2}$ & $u_{1, r m s}$ \\
0 & - & 122 & 120 & 0.011 & - & 12 & 15 & 2 & 1 & 1.26 \\
25 & 2 & 127 & 199 & 0.024 & 5 & 1.3 & 1.95 & 2 & 1 & 1.42 \\
25 & 6 & 126 & 172 & 0.019 & 10 & 1.5 & 1.8 & 2 & 1 & 1.23 \\
25 & 10 & 125 & 164 & 0.018 & 13.5 & 1.55 & 1.85 & 2 & 1 & 1.22 \\
25 & 14 & 125 & 157 & 0.017 & 16.5 & 1.8 & 1.85 & 2 & 1 & 1.15 \\
25 & 18 & 125 & 137 & 0.016 & 19 & 1.6 & 1.9 & 2 & 1 & 1.22 \\
50 & 2 & 123 & 208 & 0.025 & 4.3 & 1.3 & 1.5 & 3 & 1 & 1.30 \\
50 & 6 & 122 & 208 & 0.019 & 10 & 1.2 & 1.55 & 2 & 1 & 1.27 \\
50 & 10 & 122 & 189 & 0.017 & 13.5 & 1.5 & 1.6 & 2 & 1 & 1.17 \\
50 & 14 & 122 & 180 & 0.016 & 16.5 & 1.45 & 1.85 & 2 & 1 & 1.25 \\
50 & 18 & 122 & 150 & 0.016 & 18.5 & 1.45 & 1.6 & 2 & 1 & 1.18 \\
100 & 2 & 123 & 257 & 0.025 & 3.5 & 1.1 & 1.5 & 4 & 1 & 1.44 \\
100 & 6 & 122 & 232 & 0.020 & 7.5 & 1.15 & 1.35 & 4 & 1 & 1.28 \\
100 & 10 & 121 & 220 & 0.018 & 10.5 & 1.3 & 1.35 & 4 & 1 & 1.21 \\
100 & 14 & 122 & 220 & 0.017 & 12.5 & 1.25 & 1.35 & 3 & 1 & 1.21 \\
100 & 18 & 120 & 209 & 0.016 & 14.5 & 1.35 & 1.45 & 3 & 1 & 1.20
\end{tabular}

TABLE 3. Summary of fitting parameters in the model spectrum $E^{M}(\kappa)$ (equation (4.9)) at five times for each of the three investigated PEO concentrations. Here $\eta_{0}$ is obtained with the solvent viscosities $v_{s}$ of table 2 and $\eta$ from equation (4.5). In all cases $\Gamma=1$ and $\gamma=20$.

(i) the noise is by far the most dominant contributor to the spectral corrections;

(ii) the spectral fits made possible by (4.9) are of high quality, despite a marginal inertial range in the polymer spectrum;

(iii) this high quality is only possible because the parameters in $\mathscr{F}_{4}^{M}(4.9 b)$ were determined individually for $E_{11}$ and $E_{22}$ to account for the slight anisotropy of 'our' turbulence at low wavenumbers; in particular, the turbulence energy injected into the flow by the beaded grid was consistently distributed over a broader range of wavenumbers in the $x_{2}$ direction than in the $x_{1}$ direction;

(iv) beyond the injection scale the isotropy of the turbulence is excellent;

(v) the spectral region of primary interest, where the Kolmogorov inertial range gives way to the elastic range, is only weakly affected by the spectral corrections, except near the end of experimental runs.

Also included in figure $5(b)$ are estimates of the statistical error near the Lumley and viscous scales. The widths of the error boxes represent the horizontal shift between spectra corrected according to the first and the second right-hand side of (2.6). The vertical extensions of the error boxes represent the $95 \%$ confidence interval for the average over 100 samples. This error measure was chosen because it closely corresponds to the vertical spread of the independently averaged spectra in figures 6 and 7 below the respective Lumley scales.

The central results of the present study are collected in figures 6 and 7 which show velocity spectra averaged over time intervals of 100-300 s (interval centred on 2 laps after the start), 300-500 s (4 laps) and so forth. The principal fitting parameters for the corresponding model spectra $E^{M}$ (equation (4.9)) fitted to the experimental spectra are compiled in table 3 . The parameter of most interest, the Lumley scale $\kappa_{L} \equiv 1 / \ell_{L}$, and its time evolution are discussed in the following section. 


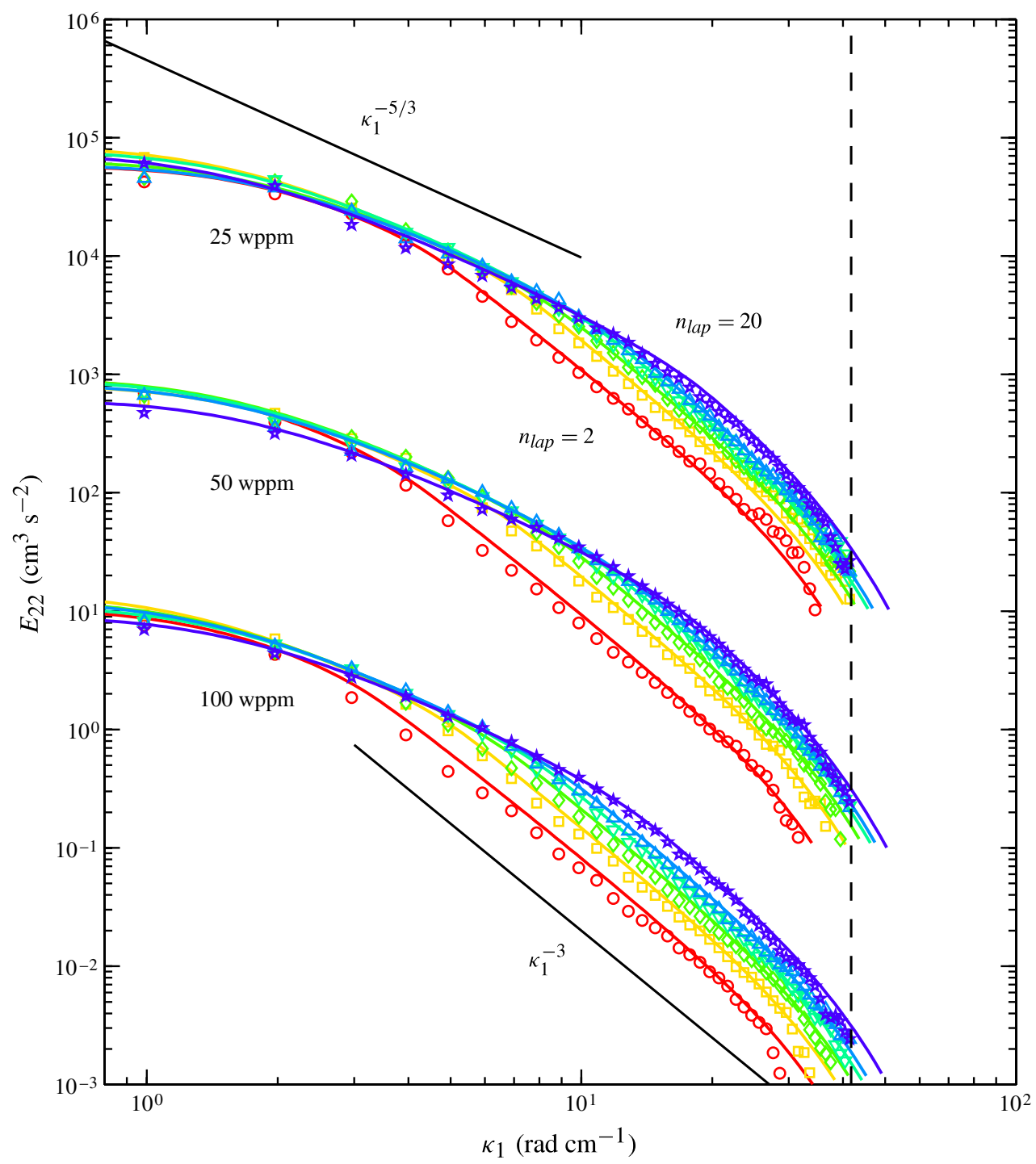

FIGURE 7. Fully corrected transverse velocity spectra. See the caption of figure 6 for the legend.

\section{Temporal evolution of the Lumley scale}

To obtain a consistent time evolution of the break point between the $-5 / 3$ and -3 ranges in the three-dimensional spectrum, i.e. the 'Lumley' wavenumber $\kappa_{L}$, two approaches were used. The first was to directly use the fitted $\kappa_{L}$ from the threedimensional model spectrum $E^{M}(\kappa)$ (4.9) used to correct the spectra $E_{i i}\left(\kappa_{1}\right)$ (cf. $\S 2.3$ ). The second was to determine the intersection of $-5 / 3$ and -3 power laws fitted individually to $E_{11}\left(\kappa_{1}\right)$ and $E_{22}\left(\kappa_{1}\right)$ and to use (4.10).

The Lumley lengths obtained from figures 6 and 7 are plotted in figure $8(a)$ versus the number of laps, $n_{\text {lap }}$, the fluid goes around the tunnel. This measure of time indicates directly the number of times the fluid passes through the grid and the 

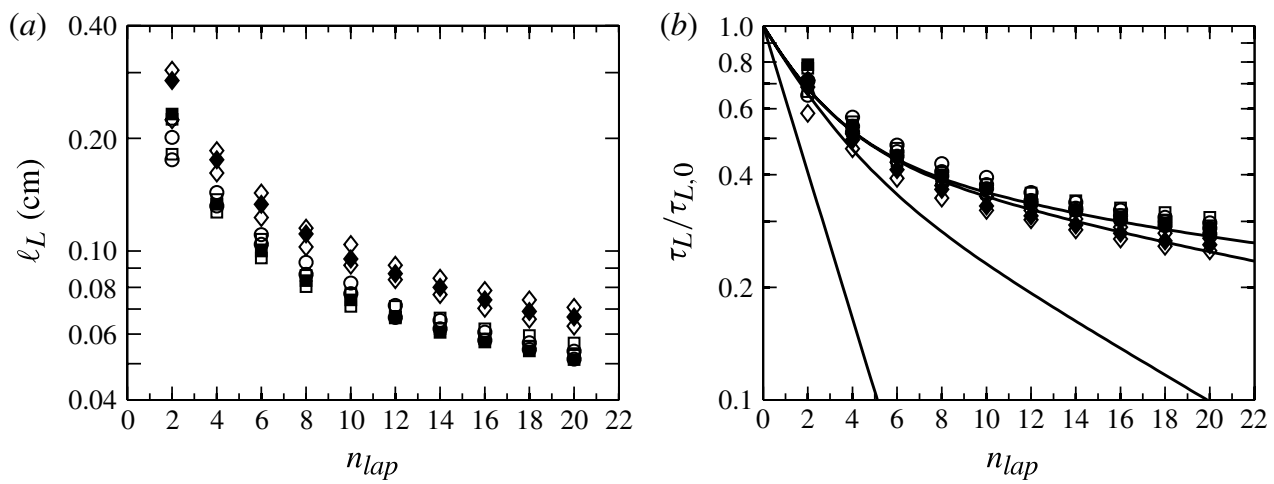

FIGURE 8. (a) Dimensional Lumley lengths $\ell_{L} \equiv 1 / \kappa_{L}$ versus $n_{\text {lap }}$ (laps around the facility; 1 lap $=100 \mathrm{~s})$ for $25 \mathrm{wppm}(\bigcirc, \boldsymbol{\bullet}), 50 \mathrm{wppm}(\square, \mathbf{\square})$ and $100 \mathrm{wppm}(\diamond, \diamond)$. Determination from longitudinal and transverse spectra (open symbols) according to (4.10); directly from the fitted model profile (4.9) (solid symbols). (b) Plot normalized according to (6.3). The solid lines illustrate the cascade model $M_{\mu}\left(n_{\text {lap }}\right) / M_{\mu}(0)((6.1)$ and (6.2)) with, from bottom to top, the contribution of the initial molecular weight $M_{\mu}$, of $M_{\mu}$ and $M_{\mu} / 2$, of $M_{\mu}, M_{\mu} / 2$ and $M_{\mu} / 4$, and of the complete cascade.

pump which are mainly responsible for the mechanical degradation of the PEO. On the grid bars, the rate of elongational strain near the upstream stagnation lines was $\sim 1.6 \times 10^{3} \mathrm{~s}^{-1}$, while the highest wall shear rate was $\sim 4 \times 10^{4} \mathrm{~s}^{-1}$, enough to cause scission of the PEO molecules (see, e.g., Odell \& Keller 1986; Sim, Khomami \& Sureshkumar 2007). For the honeycomb and the pump, we do not have the necessary flow information to estimate shear rates, but it is safe to assume that they are locally at least as high as on the grid bars.

To model the temporal decrease of $\ell_{L}$ from its initial value $\ell_{L}\left(n_{\text {lap }}=0\right)$ due to mechanical polymer degradation, a cascade model is proposed here. Since $M_{\mu}$ is an effective or average molecular weight, we assume that mechanical tearing of the original molecules produces on average molecules with $M_{\mu} / 2$ which in turn are torn into pieces of $M_{\mu} / 4$, etc. (see, e.g., Odell \& Keller 1986). The mass fractions $m_{i}$ of polymers with $M_{\mu} / 2^{i}$ are modelled by the cascade

$$
m_{i}= \begin{cases}\exp \left(-0.45 n_{\text {lap }}\right) & \text { for } i=0 \\ {\left[1-\sum_{j=0}^{i-1} m_{j}\right] \exp \left(-0.45 \times 0.18^{i} n_{\text {lap }}\right)} & \text { for } i>0\end{cases}
$$

where the exponent $-0.45 \times 0.18^{i}$ is the rate of depletion of mass fraction $m_{i}$ which becomes progressively smaller with $i$. The resulting time-dependent average molecular weight is then simply

$$
M_{\mu}\left(n_{\text {lap }}\right)=M_{\mu}\left(n_{\text {lap }}=0\right) \sum_{i=0}^{\infty} \frac{m_{i}}{2^{i}} .
$$

It is evident from figure $8(a)$ that the Lumley lengths $\ell_{L}\left(n_{\text {lap }}\right)$ for 25 and $50 \mathrm{wppm}$ coincide within experimental error, while the $\ell_{L}$ for $100 \mathrm{wppm}$ are larger. This suggests a collapse of Lumley lengths in a way similar to the Rouse-Zimm model 
for the polymer time constant $\tau$ (see (3.4)):

$$
\ell_{L}\left(n_{\text {lap }}\right) \equiv \alpha^{3 / 4} \epsilon_{0}^{1 / 2} \tau_{L}^{3 / 2} \quad \text { with } \tau_{L}\left(n_{\text {lap }}\right)=4.61 \times 10^{4} v_{s} \frac{M_{\mu}\left(n_{\text {lap }}\right)}{R T},
$$

where $\alpha=3 / 2$ is the Kolmogorov prefactor for the three-dimensional inertial range spectrum. Note that, in order to match the present experimental evidence, the prefactor in (6.3) has been made much larger than in (3.4) (see figure 3) and the dependence of $\tau_{L}$ on $M_{\mu}$ made weaker than in the Rouse-Zimm model by replacing $\mu_{s}[\mu]$ by the kinematic viscosity of the solvent.

The Lumley time constants $\tau_{L}$ obtained from the $\ell_{L}$ of figure $8(a)$ and normalized according to (6.3) with $M_{\mu}\left(n_{\text {lap }}=0\right)$ are displayed in figure $8(b)$. Tables 2 and 3 give the initial $M_{\mu}$ and $\epsilon_{0}$ for the different concentrations, respectively $\left(\epsilon_{0}(t=100 \mathrm{~s})\right.$ was used for $\left.\epsilon_{0}(t=0)\right)$. While the collapse of the normalized $\tau_{L}$ is within the estimated uncertainty of $\pm 15 \%$ the normalized time constant for $100 \mathrm{wppm}$ still appears to be lower than the $\tau_{L}$ for the lower concentrations. However, with the present data it is not possible to determine whether this deviation is systematic or not. Nevertheless, the cascade model $((6.1)-(6.3))$ is shown in figure $8(b)$ to provide a good fit to the data.

\section{Outlook}

The present experiments have allowed a first look at the time evolution of turbulence spectra in polymer solutions which reveal, we believe for the first time, a clear but time-dependent Lumley scale beyond which elastic effects lead to an energy decay $E \propto \kappa^{-3}$. The observed time dependence of the Lumley scale has been linked to polymer degradation. These findings are in accord with the early speculation of Hinch Hinch (1977) and Hinch \& Elata (1979) that polymers act as a rate of strain limiter as long as they are much shorter than the viscous scale. The findings also imply a collective behaviour of individual polymer molecules, as noted by De Angelis et al. (2002), which may be brought about by sharp flow accelerations on the scale of turbulence 'eddies'.

The experiments can and should of course be improved: a larger turbulence Reynolds number, i.e. a larger dissipation rate $\epsilon_{0}$, would increase the different scaling ranges and permit a more accurate determination of spectral slopes. However, this would require a correspondingly larger dynamic range of the PIV equipment and polymer degradation is likely to be aggravated. Note that the present set-up was relatively well-matched to the Reynolds number, as the major spectral corrections did not significantly affect the determination of the Lumley scale $\ell_{L}$ (see figure 5).

It would be even more important to continue the experiments with progressively lower PEO concentrations to identify the critical concentration $c_{\text {crit }}$ corresponding to $\Gamma=1$ (see the paragraph following equation (4.2)) which separates the strong back reaction regime discussed for instance by Balkovsky et al. (2001) and Fouxon \& Lebedev (2003), in which the elastic range extends all of the way to the viscous dissipation range, from the regime with $c<c_{c r i t}$. Such experiments would reveal the parameter dependence of $c_{c r i t}$ and, more importantly, test available models, including our ansatz of equation (4.2) with the $\kappa$-independent factor $\Gamma$. Running experiments with very low concentrations would in particular allow testing of the model feature of two inertial ranges separated by an elastic range for $0<\Gamma<1$. As already noted in $\S 4$, such spectra strongly resemble the spectra of Benzi et al. (2003) obtained from shell models. However, the Reynolds number of the present experiments is too low to determine the conditions leading to a second $-5 / 3$ range after the elastic range. 
Answers to the above questions would likely advance the understanding of the effect of polymers on wall-bounded turbulent flows, i.e. on polymer drag reduction (see, e.g., Sreenivasan \& White 2000). In particular it would become possible to estimate the critical polymer concentration necessary for the maximum reduction of the peak turbulent strain rate in the boundary layer. Furthermore, the present model may provide a good estimate of the local effect of polymers and in turn allow the separation of the local effect from the indirect effects on dynamic larger-scale (instability) processes due to the modified mean flow profile. Finally, it may be interesting to explore a numerical scheme which limits the instantaneous local rate of strain with a body force, updated at every time step, to represent the bulk effect of polymers.

\section{Acknowledgement}

This work has benefitted from the financial support by the Swiss National Research Fund (SNF) under grant no. 200020-119824.

\section{REFERENCES}

Balkovsky, E., Fouxon, A. \& Lebedev, V. 2001 Turbulence of polymer solutions. Phys. Rev. E 64, 056301.

BARnARD, B. J. S. \& SEllin, R. H. J. 1969 Grid turbulence in dilute polymer solutions. Nature 222 (5199), 1160-1162.

Benzi, R., De Angelis, E., Govindarajan, R. \& Procaccia, I. 2003 Shell model for drag reduction with polymer additives in homogeneous turbulence. Phys. Rev. E 68 (1), 016308.

Berti, S., Bistagnino, A., Boffetta, G., Celani, A. \& Musacchio, S. 2006 Small-scale statistics of viscoelastic turbulence. Europhys. Lett. 76 (1), 63-69.

Brostow, W. 2008 Drag reduction in flow: review of applications, mechanism and prediction. J. Ind. Engng Chem. 14 (4), 409-416.

De Angelis, E., Casciola, C. M., Benzi, R. \& Piva, R. 2005 Homogeneous isotropic turbulence in dilute polymers. J. Fluid Mech. 531, 1-10.

De Angelis, E., Casciola, C. M. \& Piva, R. 2002 DNS of wall turbulence: dilute polymers and self-sustaining mechanisms. Comput. Fluids 31, 495-507.

Den Toonder, J. M. J., DraAd, A. A., Kuiken, G. D. C. \& Nieuwstadt, F. T. M. 1995 Degradation effects of dilute polymer-solutions on turbulent drag reduction in pipe flows. Appl. Sci. Res. 55 (1), 63-82.

Den Toonder, J. M. J., Hulsen, M. A., Kuiken, G. D. C. \& Nieuwstadt, F. T. M. 1997 Drag reduction by polymer additives in a turbulent pipe flow: numerical and laboratory experiments. J. Fluid Mech. 337, 193-231.

Elghobashi, S. 1994 On predicting particle-laden turbulent flows. Appl. Sci. Res. 52 (4), 309-329.

Escudier, M. P., Presti, F. \& SMith, S. 1999 Drag reduction in the turbulent pipe flow of polymers. J. Non-Newtonian Fluid 81 (3), 197-213.

Ferry, J. D. 1980 Viscoelastic Properties of Polymers, 3rd edn. Wiley.

Foucaut, J. M., Carlier, J. \& Stanislas, M. 2004 PIV optimization for the study of turbulent flow using spectral analysis. Meas. Sci. Technol. 15, 1046-1058.

Fouxon, A. \& Lebedev, V. 2003 Spectra of turbulence in dilute polymer solutions. Phys. Fluids 15 (7), 2060-2072.

Friehe, C. A. \& SCHWARZ, W. H. 1970 Grid-generated turbulence in dilute polymer solutions. J. Fluid Mech. 44, 173-193.

GADD, G. E. 1965 Turbulence damping and drag reduction produced by additives in water. Nature 206 (4983), 463-467.

Graessley, W. W. 1980 Polymer-chain dimensions and the dependence of viscoelastic properties on concentration, molecular-weight and solvent power. Polymer 21 (3), 258-262. 
Groisman, A. \& Steinberg, V. 2000 Elastic turbulence in a polymer solution flow. Nature 405 (6782), 53-55.

Groisman, A. \& Steinberg, V. 2004 Elastic turbulence in curvilinear flows of polymer solutions. New J. Phys. 6, 29.

HiNCH, E. J. 1994 Uncoiling a polymer molecule in a strong extensional flow. J. Non-Newtonian Fluid 54, 209-230.

Hinch, E. J. 1977 Mechanical models of dilute polymer solutions in strong flows. Phys. Fluids 20 (10), 22-30.

Hinch, E. J. \& ElATA, C. 1979 Heterogeneity of dilute polymer solutions. J. Non-Newtonian Fluid 5, 411-425.

Kalashnikov, V. N. \& Vlasov, S. A. 1978 Scale-dependent effect in laminar-flow of dilute polymer-solution in tubes. Rheol. Acta 17 (3), 296-302.

KIM, K. \& SiRviente, A. I. 2007 Wall versus centreline polymer injection in turbulent channel flows. Flow Turbul. Combust. 78 (1), 69-89.

Lavoie, P., Avallone, G., De Gregorio, F., Romano, G. P. \& Antonia, R. A. 2007 Spatial resolution of PIV for the measurement of turbulence. Exp. Fluids 43 (1), 39-51.

Liberzon, A., Guala, M., Kinzelbach, W. \& Tsinober, A. 2006 On turbulent kinetic energy production and dissipation in dilute polymer solutions. Phys. Fluids 18 (12), 125101.

Liberzon, A., Holzner, M., Luthi, B., Guala, M. \& Kinzelbach, W. 2009 On turbulent entrainment and dissipation in dilute polymer solutions. Phys. Fluids 21 (3), 035107.

Lumley, J. L. 1964 Turbulence in non-Newtonian fluids. Phys. Fluids 7 (3), 335-337.

Lumley, J. L. 1969 Drag reduction by additives. Annu. Rev. Fluid Mech. 1, 367-384.

Lumley, J. L. 1973 Drag reduction in turbulent flow by polymer additives. J. Polym. Sci. Macrom. Rev. 7, 263-290.

McСомв, W. D. 1990 The Physics of Fluid Turbulence. Oxford University Press.

McСomb, W. D., Allan, J. \& Greated, C. A. 1977 Effect of polymer additives on the small-scale structure of grid-generated turbulence. Phys. Fluids 20 (6), 873-879.

Mejia-Alvarez, R. \& Christensen, K. T. 2011 Polymer-induced turbulence modifications in an impinging jet. Exp. Fluids 1-24.

Morgan, D. T. G. \& Pike, E. W. 1972 Influence of molecular weight upon drag reduction by polymers. Rheol. Acta 11 (2), 179-184.

Morrison, F. A. 2001 Understanding Rheology. Oxford University Press.

Odell, J. A. \& Keller, H. H. 1986 Flow-induced chain fracture of isolated linear macromolecules in solution. J. Polym. Sci. Part B: Polym. Phys. 24, 1889-1916.

Ouellette, N. T., Xu, H. \& Bodenschatz, E. 2009 Bulk turbulence in dilute polymer solutions. J. Fluid Mech. 629 (1), 375-385.

Patterson, G. K., Zakin, J. L. \& Rodrigue, J. M. 1969 Drag reduction; polymer solutions soap solutions and solid particle suspensions in pipe flows. Ind. Engng Chem. 61 (1), 22-30.

PIPE, C. J. (2005) Experiments investigating the effects of fluid elasticity on laminar vortex shedding from a cylinder. PhD thesis, EPFL.

Poelma, C., Westerweel, J. \& Ooms, G. 2006 Turbulence statistics from optical whole-field measurements in particle-laden turbulence. Exp. Fluids 40 (3), 347-363.

Pope, S. B. 2008 Turbulent Flows, 5th edn. Cambridge University Press.

Raffel, M., Willert, C., Wereley, S. \& Kompenhans, J. 2007 Particle Image Velocimetry: a Practical Guide. Springer.

Rodd, L. E., Cooper-White, J. J., Boger, D. V. \& MCKinley, G. H. 2007 Role of the elasticity number in the entry flow of dilute polymer solutions in micro-fabricated contraction geometries. J. Non-Newtonian Fluid 143 (2-3), 170-191.

Rozhkov, A., Prunet-Foch, B. \& Vignes-AdLer, M. 2003 Impact of drops of polymer solutions on small targets. Phys. Fluids 15 (7), 2006-2019.

Sellin, R. H. J., Hoyt, J. W., Pollert, J. \& Scrivener, O. 1982 The effect of drag reducing additives on fluid-flows and their industrial applications. 2. Present applications and future proposals. J. Hydraul. Res. 20 (3), 235-292. 
Sim, H. G., Khomami, B. \& Sureshrumar, R. 2007 Flow-induced chain scission in dilute polymer solutions: algorithm development and results for scission dynamics in elongational flow. J. Rheol. 51, 1223-1251.

Sreenivasan, K. R. \& White, C. M. 2000 The onset of drag reduction by dilute polymer additives, and the maximum drag reduction asymptote. J. Fluid Mech. 409, 149-164.

Sureshimmar, R., Beris, A. N. \& Handler, R. A. 1997 Direct numerical simulation of the turbulent channel flow of a polymer solution. Phys. Fluids 9, 743-755.

SylVester, N. D. \& TYleR, J. S. 1970 Dilute solution properties of drag-reducing polymers. Ind. Eng. Chem. Prod. Res. Develop. 9 (4), 548-553.

TenneKes, H. \& Lumley, J. L. 1972 A First Course in Turbulence. MIT.

Tirtaatmadja, V., McKinley, G. H. \& Cooper-White, J. J. 2006 Drop formation and breakup of low viscosity elastic fluids: effects of molecular weight and concentration. Phys. Fluids $\mathbf{1 8}$ (4), 043101.

Toms, B. A. 1948 The flow of linear polymer solutions through straight tubes at large Reynolds numbers. In Proceedings of the First International Congress on Rheology, vol. 2, p. 135. North-Holland Publishing Company.

VAn Doorn, E., White, C. M. \& Sreenivasan, K. R. 1999 The decay of grid turbulence in polymer and surfactant solutions. Phys. Fluids 11 (8), 2387-2393.

VIRK, P. S. 1975 Drag reduction fundamentals. AIChE J. 21 (4), 625-656.

Virk, P. S., Merill, E. W., Mickley, H. S., Smith, K. A. \& Mollo-Christensen, E. L. 1967 The Toms phenomenon: turbulent pipe flow of dilute polymer solutions. J. Fluid Mech. 30, 305-328.

Vonlanthen, R. (2010) The effects of fluid elasticity on grid turbulence. PhD thesis, EPFL.

Vonlanthen, R. \& Monkewitz, P. A. 2011 A novel tethered-sphere add-on to enhance grid turbulence. Exp. Fluids 51, 579-585.

Warholic, M. D., Massah, H. \& Hanratty, T. J. 1999 Influence of drag-reducing polymers on turbulence: effects of Reynolds number, concentration and mixing. Exp. Fluids 27 (5), $461-472$.

Westerweel, J. \& Scarano, F. 2005 Universal outlier detection for PIV data. Exp. Fluids 39 (6), 1096-1100.

WyngaARD, J. C. 1968 Measurement of small-scale turbulence structure with hot wires. J. Phys. E Sci. Instrum. 1 (11), 1105-1108. 